\title{
Noticias telúricas: el "temblor grande" de 1822 a través de la prensa chilena y extranjera ${ }^{1}$
}

\section{Nicolás Gorigoitía Abbott ${ }^{2}$ Alfredo Palacios Roa ${ }^{3}$}

Pestes, inundaciones, sequías y terremotos; estos y otros fenómenos, provocados por los riesgos naturales, han acompañado la formación y los distintos procesos históricos de Chile como reino y nación. Aquella tierra "mal infamada" de la que hablaban los primeros conquistadores y cronistas siempre estuvo ligada en alguna dimensión con lo catastrófico, como un devenir infausto que, en palabras de Rolando Mellafe, generó un carácter telúrico en su población. A inicios del siglo XIX, la formación de la república y el Estado independiente también convivieron con eventos naturales, cuyas consecuencias fueron determinadas por el contexto que se vivía en la época, siendo el terremoto del 19 de noviembre de 1822 uno de los más paradigmáticos, tanto por sus efectos como por la compleja trama de la cual terminó siendo parte.

A pesar del extenso listado de sismos que acumulaba hasta entonces Chile a lo largo de su historia colonial y republicana temprana, el acontecimiento de 1822 se destaca dentro de esa lista, no tanto por sus características ni por la destrucción causada -modesta en comparación con otros terremotos-, sino más bien por la información que emanó. A los informes oficiales, relaciones de sucesos y material epistolar propio de estos eventos, se sumaron notas periodísticas y noticias que informaron a la ciudadanía chilena y extranjera sobre los hechos acaecidos esa noche de noviembre en Valparaíso, Santiago y alrededores. A través de esos impresos se dio a conocer la situación de las ciudades afectadas, así como las diligencias y respuestas que el primer gobierno republicano emprendió para dar salida a este estado de calamidad. En estos escritos se aprecia la transición que se vivía en aquella época bisagra, pasando de una sociedad colonial, con un fuerte acento religioso, a una

1 Este trabajo forma parte del proyecto FONDECYT Iniciación No 11160157, "Impacto sociopolítico de los temblores y terremotos en Chile a lo largo del siglo XIX", a cargo de Alfredo Palacios Roa.

2 Chileno. Máster en Historia, Universidad Jaume I, España. E-mail: nicolas.gorigoitia.a@gmail.com

3 Chileno. Doctor en Historia, Universidad de Sevilla, España. Académico del Departamento de Historia y Ciencias Sociales, Facultad de Artes Liberales, Universidad Adolfo Ibáñez, Chile. E-mail: alfredo.palacios@uai.cl 
republicana y laica, cuestión materializada en la mixtura en los relatos que incluyen desde la noción de "castigo divino" hasta observaciones científicas del fenómeno como evento natural.

El conjunto de notas periodísticas transcritas a continuación constituye el corpus noticioso del terremoto de 1822. Si bien es una entrega pequeña en cantidad, cualitativamente tienen una enorme importancia, tanto por las razones esgrimidas en el párrafo anterior como por ser ejemplificadoras de los medios disponibles en la época. A su vez, dan cuenta de cómo un evento se transforma en noticia, generando una dinámica de circulación de la información la cual, si bien a mediados del siglo XIX se instituirá como la norma, hasta el momento era inédita.

\section{El terremoto de 1822 en su contexto}

Hacia finales de 1822 la situación en Chile era compleja. Las circunstancias económicas y productivas propias de un país en construcción que venía saliendo de numerosos enfrentamientos bélicos, más la suma de las tensiones sociopolíticas de un tejido social que enfrenta el desafío de su adecuación desde una realidad monárquica a una republicana, fueron el telón de fondo del primer gobierno de hecho y derecho de Chile, con Bernardo O'Higgins a la cabeza como su director supremo. Tal cargo, que ostentó entre 1817 y 1823, fue obtenido por su destacado desempeño militar en la lucha contra las huestes de la monarquía hispana, así también por el respeto y cercanía que tenía con José de San Martín, sindicado como el autor intelectual de ésta y otras victorias americanas contra el gigante europeo.

Ciertamente, el rioplatense fue la primera opción de la elite santiaguina para convertirse en la máxima autoridad del país, pero este rechazó dicho ofrecimiento en favor de O'Higgins, apelando a su deber de continuar con la liberación del continente, así como por la estrecha amistad que compartía con militar chillanejo. Ahora, si bien San Martín respaldó públicamente la elección de O'Higgins, inicialmente había propuesto nombrar tres electores -uno por cada provincia- para sancionar la designación del supremo director (Barros Arana, 1857, III: 444); sin embargo, el cabildo capitalino, aduciendo la necesidad de tener una cabeza política y militar en tal vulnerable contexto, rápidamente nombró a O'Higgins en febrero de 1817 (Gorigoitía, 2016: 190). Aquella situación vino a generar uno de los primeros roces de la elite santiaguina con las regionales en la época republicana, ya que este último grupo consideró que el mecanismo para nombrar y ratificar al primer mandatario presentó una suerte de "vicio de origen" (Cartes, 2014: 265). 
Esas primeras tensiones surgidas entre las elites y el director supremo fueron aumentando con las políticas implementadas durante su gobierno, sobre todo con las relacionadas a los ámbitos culturales, sociales y económicos. En efecto, y a pesar de las victorias militares que ratificaron de facto el poder de O'Higgins y la independencia de Chile, el ímpetu reformista del primer gobierno republicano incomodó de sobremanera a los sectores más conservadores de la sociedad chilena, los cuales se resistían a asumir el paso de un régimen monárquico a uno republicano y "secular". ${ }^{4}$ Medidas tales como la abolición de títulos nobiliarios, distintivos y escudos de armas generaron un fuerte rechazo en esos grupos, especialmente entre los capitalinos. Del mismo modo, la prohibición de actividades como las riñas de gallos y las corridas de toros hicieron que el pueblo también comenzara a demostrar su molestia por la "avanzada" del gobierno (Goldman, 2003, V: 204). Así también, la supresión de algunas procesiones religiosas y la idea de fundar un cementerio para los disidentes provocaron una fuerte resistencia en altos miembros de la Iglesia Católica, tensión que se agudizó tras la decisión del gobierno de mantener el derecho de patronato, cuestión que la Iglesia rechazaba rotundamente.

Del mismo modo, los representantes regionales comenzaron a demostrar su inconformidad con la línea de acción de O'Higgins, sobre todo en algunos temas económicos y políticos. Por ejemplo, uno de estos fue el complejo momento financiero que se vivía en algunas regiones a raíz de los gastos provocados por la guerra, y el segundo estaba en directa relación con el excesivo poder del que se iba haciendo el Director Supremo considerado con rasgos dictatoriales y centralistas. En consecuencia, el contraste entre la situación del sur, donde la escases de víveres y el retraso en los sueldos militares era lamento de todos los días, sumado al apoyo económico que recibían las campañas militares lideradas por San Martín en las costas americanas, incitaron sendas quejas por parte de diversas autoridades locales, acusando manipulación por parte del trasandino y poca preocupación de O’Higgins por la situación interna.

Esas mismas personalidades, junto con las elites locales, veían con desconfianza las definiciones políticas del Director Supremo, especialmente aquellas relacionadas con su propia figura, acusándolo de querer fragmentar el poder regional en favor del suyo. Esto último fundamentado en las amplias atribu-

4 A pesar de la desconfianza de los sectores conservadores en relación al vínculo de los regímenes republicanos y el laicismo, desde la primera Constitución Política chilena se estableció la titularidad de la religión católica, apostólica y romana, que en el caso de la era o'higginista se constituyó como "única" (Proyecto de Constitución Provisoria para el Estado de Chile, 1818: 8) y "con exclusión de cualquier otra" (Constitución Política del Estado de Chile, 1822: 4). 
ciones que se establecieron para la primera autoridad nacional en la carta magna provisoria de 1818 (como, por ejemplo, dirigir la Armada, nombrar funcionarios públicos, manejar las arcas fiscales y las relaciones exteriores), y que según sus detractores fueron profundizadas en la nueva Constitución de 1822, al designar directamente a O'Higgins por seis años más en su cargo con opción de reelección por cuatro más. En este mismo contexto, también se reestructuró geográficamente la administración política, creando ocho departamentos en reemplazo de las tres antiguas provincias, despertando suspicacias especialmente en Concepción al ser interpretadas como una forma de dividir la provincia "para quitarle su antigua y tradicional importancia" (Barros Arana, 1894, XIII: 359).

Igualmente, y de forma paralela a la firma del nuevo reglamento constitucional, el gobierno promulgó una ordenanza comercial que inspiró la misma o mayor desconfianza que la carta magna, ya que se instauraba a Valparaíso como puerto franco y principal del país, en desmedro de los otros embarcaderos, como los de Coquimbo y Talcahuano. En este contexto, y a juicio de María Graham (que residía en el país en aquellos años), con aqueIla jurisprudencia se hacía una "injusticia manifiesta, fuera de las enormes imprudencias de semejante declaración, a causa de los antiguos celos de los puertos del sur y los que en diversas ocasiones han aparecido en Coquimbo" (Graham, 1953: 198).

Las imprudencias y celos mencionados por la reconocida viajera inglesa tenían directa relación con todos los desencuentros entre la administración de O'Higgins y los distintos actores políticos de la época. En ello jugó un rol preponderante el círculo de confianza del director supremo, en especial con su ministro de Hacienda y mano derecha, José Antonio Rodríguez Aldea, por su supuesto pasado realista y manejos impropios durante su gestión en el gabinete. También, la cercanía con San Martín le generó problemas, tanto por ser sindicado como el poder tras las sombras de O'Higgins como por el uso de las naves chilenas en las campañas peruanas. Lord Thomas Cochrane, vicealmirante y encargado de la formación de la Marina Nacional, mantuvo una tensa relación con el libertador americano por el uso de tales naves, que consideraba indebido; de hecho, en sus memorias criticó a O'Higgins por el trato preferente que le dio a San Martín en su paso por Chile tras dejar el protectorado del Perú. Según el conde de Dundonald, el líder trasandino debería haber sido apresado por sus ilícitos actos tanto en Chile como en Perú; sin embargo, la actitud del gobierno chileno fue todo lo contrario, alojando a San Martín en el palacio "y los ministros le tribularon todo género de atenciones... una conducta tal en el gobierno [que] produjo un gran descontento en el público" (Cochrane, 1863: 270). Aquel descontento también fue descrito por María Graham, avizorando un mal desenlace para O'Higgins y su gestión, ya que según su observación: 
"De norte y del sur llegan noticias y rumores de descontentos de diversas especies. Los hermanos y parientes de muertos y de los proscriptos que no los han olvidado, ven con dolor y cólera colmado de honores al hombre que consideran autor de sus infortunios. Respetando la persona del director, ven en él al amigo y aliado de San Martín, al sostenedor de Rodríguez y sus compañeros, y ya me parece oír ese murmullo sordo y encubierto que precede a la guerra civil" (Graham, 1953: 209).

En aquel escenario, lleno de incertidumbre, O'Higgins decidió trasladarse a Valparaíso para solucionar a lo menos uno de sus problemas: la deuda adquirida con los integrantes de los buques navales por sus sueldos impagos. Con setenta mil pesos a cuesta, obtenidos a través de un préstamo con una casa comercial santiaguina, el supremo director pretendía restituir las confianzas con Lord Cochrane y su gente; sin embargo, el martes 19 de noviembre de 1822, y "sin anteceder ruido ni otra señal precursora" (Miquel, 1859: 299), la tierra del puerto comenzó a sacudirse violentamente, cambiando drásticamente los planes de O'Higgins.

Durante esa jornada, y cuando el reloj marcaba las diez y media de la noche (Palacios, 2015: 138), la zona central de Chile experimentó un sismo de gran intensidad, que estudios científicos recientes han calibrado entre 8 y 8.5 Mw (Lomnitz, 2004: 372; Barrientos, 2007: 273). Ahora, si bien su epicentro se situó en áreas cercanas a La Ligua, el foco de atención fue Santiago, en cuanto la capital del país, y Valparaíso, como su centro administrativo y comercial más cercano. En esta última ciudad se destruyeron la mayoría de los edificios público (casa de gobierno, cárcel y cuartel, Aduana y sus almacenes, edificio de correos y hospitales, entre otros), la totalidad de los templos religiosos y más de setecientas casas particulares, que, junto a la generación de hendiduras en el suelo, producción de "brotes de agua" ${ }^{5}$ en el sector del Almendral y notorios accesos y recesos del mar, ${ }^{6}$ generaron -a decir de un testigo- un verdadero "concierto terrorífico" (Longeville, 1923: 49). Con todo, y a pesar de la enorme destrucción en este reconocido conjunto urbano (figura 1), solamente se registraron setenta y dos víctimas y ciento diez heridos, entre ellos el mismo Bernardo O'Higgins (Sepúlveda, 2007:

5 Este proceso, conocido como licuefacción del suelo, es originado por el reacomodamiento de las partículas sólidas (granos) como resultado de las vibraciones producidas por un sismo. Al reducirse los espacios se incrementa la presión de poros, por lo que la presión de contacto entre los granos disminuye y, como consecuencia, el suelo se comporta como un líquido denso (Matamoros, 1994: 17).

6 Según el informe realizado por Carlos Thurn para el gobernador de Valparaíso, las aguas del mar se elevaron hasta 12 pies (3,6 metros) de su nivel regular y se retiraron entre 8 y 10 pies (2,4 a 3 metros) "de la tierra que frecuentemente bañaba en su rivera" (Thurn, 1919: 191). 
480). Del mismo modo, en las localidades próximas al puerto, como Quintero, Limache, Quillota, Casablanca, La Ligua y Melipilla también resultaron seriamente afectadas la mayoría de sus construcciones (Palacios, 2016: 65).

Por otra parte, en la ciudad de Santiago, a pesar de haberse sentido un extraordinario estruendo "sólo se comprobaron la destrucción total o parcial de algunas casas vetustas, el desequilibrio de dos o tres torres, y algunos deterioros de fácil reparación en varios edificios públicos y particulares" (Montessus de Ballore, 1912, IV: 120) (Figura 2).

La fuerza del movimiento también se percibió en tierras trasandinas, siendo la ciudad de Mendoza una de las más sacudidas (Proctor, 1920: 55). Dentro del mismo contexto, y luego del primer movimiento, se registraron una seguidilla de violentas réplicas, las cuales se prologaron, aunque con menor intensidad y constancia, hasta el mes de abril de 1823 (Schmidtmeyer, 2014: 322).

\section{Figura 1}

El terremoto de 1822 en las calles de Valparaíso

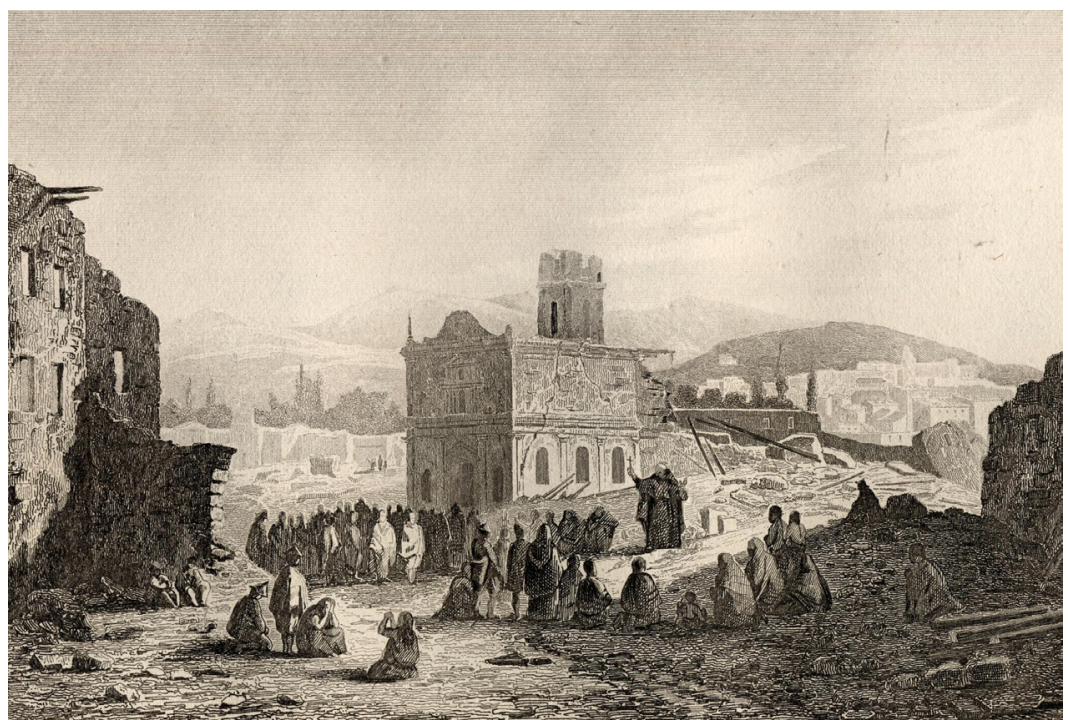

Fuente: Famín, C. (1839). Historia de Chile. Barcelona: Imprenta de Guardia Nacional, figura 19.

Tras el desastre generado por el paroxismo, no fueron pocas las personas que buscaron explicar las causas de tal funesto acontecimiento. Desde el plano espiritual, voces surgidas desde la curia católica reforzaron la tradicional idea de que tales hechos eran por causas divinas, a modo de reprimenda o llamado de atención a la población por sus pecados y mal vivir 
(Palacios, 2010: 391-392), hegemónica interpretación que dominó durante el periodo colonial, pero que desde hace pocos años había comenzado a ser refutada por distintos intelectuales chilenos de corte liberal, aduciendo la periodicidad de tales eventos naturales. El paso a una interpretación fundada y científica en relación con este tipo de fenómenos obedeció al contexto ilustrado de mediados del siglo XVIII y a experiencias europeas traumáticas pero enriquecedoras como el terremoto de Lisboa de 1755, acontecimiento clave para la promoción de nuevas posturas sobre los seísmos y sus causas naturales (Alberola, 2005: 42; Alberola, 2009: 59). En cuanto a América, el caso venezolano de marzo 1812 contribuyó en la misma dirección, abriendo el debate sobre los orígenes de los terremotos y generando un verdadero "efecto 1812" (VV. AA., 2016).

Sin ir más lejos, diez años antes del llamado "temblor grande" (Amunátegui, 1855: 431), y tomando como referencia la serie de eventos sísmicos que afectaron a Venezuela en 1812, fray Camilo Henríquez reflexionó a través de las páginas de La Aurora de Chile sobre la calidad de fenómenos de la naturaleza y sobre el origen de los sismos, asociando estos últimos fenómenos a "la inflamación de las materias combustibles, contenidas en las entrañas de la tierra"7, apartándose de las interpretaciones mítico-religiosas dominantes es aquellos años y consideradas como surgidas más del fanatismo que de la misma realidad.

7 La Aurora de Chile, Santiago de Chile, 28-I-1813: 2. 


\section{Figura 2}

El terremoto de 1822 en las calles del centro de Santiago de Chile

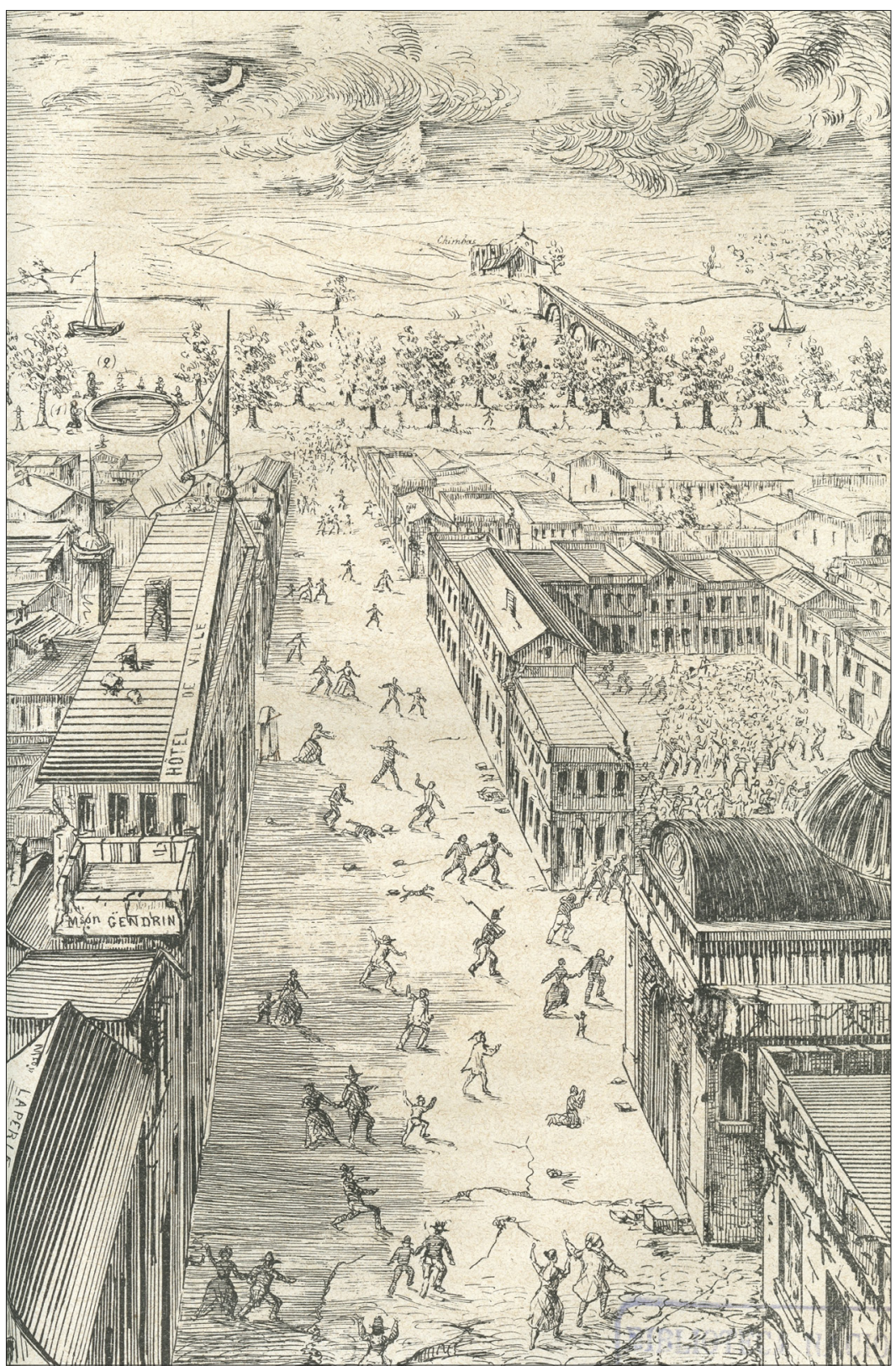

Fuente: Gendrin, V. (1856). Récit historique, exact et sincere, par mer et par terre, de quatre voyages faits au Brésil, au Chili, dans les cordilleres des Andes, a Mendoza, dans le desert, et a Buenos Aires. Versalles: Imprenta Klefer, 1856: 324-325. 
Ciertamente, el terremoto de 1822 sirvió para poner en discusión la causa de tales fenómenos; sin embargo, a pesar de que Henríquez nuevamente arremetió contra la visión tradicional (esta vez como editor del periódico El Mercurio de Chile), una avanzada conservadora insistió en atribuir, tanto el origen como las funestas consecuencias de este fenómeno, al castigo divino (Palacios, 2010: 397-399; Cid, 2014: 95-106), siendo su principal responsable el primer gobierno republicano, considerado por esta colectividad como "ateo" e "irreligioso" 8 , a pesar que una de las primeras medidas de O'Higgins fue, mediante Decreto Supremo, prohibir toda diversión pública mientras duraran los movimientos de tierra para que todos los habitantes "no tengan un embarazo que les distraiga de el objeto de elevar sus súplicas para aplacar la ira divina" (Gorigoitía, 2016: 197).

Por lo tanto, la cabeza y líder del gobierno, el general Bernando O'Higgins, hijo ilegitimo de un irlandés (O'Phelan, 2010: 101), fue sindicado como uno de los mayores culpables de tal castigo divino. Las medidas impuestas durante sus cinco años de gobierno le valió ser presa, junto a la población extranjera (especialmente estadounidenses y británicos) disidente de la "religión verdadera", y aquella transgresora de la moral y buenas costumbres, de la culpabilidad e inquina popular. ${ }^{9}$

En resumen, el descontento generalizado contra O'Higgins por su desempeño como máxima autoridad política sirvió como telón de fondo para manipular la imagen del mandatario y canalizar gran parte de la culpabilidad del terremoto en su persona. De hecho, la suma de las quejas emanadas desde elites santiaguinas y regionales, del alto clero y de un buen número de funcionarios públicos (civiles y militares), enmarcadas en un contexto altamente vulnerable debido a la emergencia que provocó del terremoto, contribuyeron a generar la atmosfera necesaria para que el mandatario, ante una eventual guerra civil, decidiera abdicar a finales de 1822, cuestión que concretó el 23 de enero de 1823 tras lo cual se exilió en el Perú (Eyzaguirre, 1950: 365). Años más tarde, en una carta particular escrita desde su hacienda de Montalván, el autodepuesto militar escribió:

8 Respecto de tal acusación, Benjamín Vicuña Mackenna señaló lo siguiente: "esta catástrofe dio lugar, sin embargo, a singulares complicaciones políticas, no por sus desastres sino por las causas que la superstición le atribuyó. La política y el fanatismo exploraron, cada cual por sus intereses, la ignorancia del vulgo echando la cual de aquel castigo del cielo, unos al mal gobierno del general O'Higgins, otros a la falta de religión de este caudillo" (Vicuña Mackenna, 1860: 460-461).

9 En efecto, en Valparaíso y Santiago se dijo que "esa ruina era el castigo por las maldades de O’Higgins que daba al pueblo libertades nunca vistas, fomentaba su instrucción y protegía a los herejes, trayendo profesores y obreros extranjeros. En Valparaíso corrieron listas para pedir la expulsión de todos ellos" (Riquelme: 1899: 366). 
"La palabra independencia, sin duda, hizo grandes cosas, pero no obró milagros y no podía disipar en un día, un mes ni un año, la corrupción e ignorancia engendrada durante tres siglos por un mal gobierno; igualmente corrompido e ignorante. De allí la debilidad, inconsistencia, el amor al cambio y el deseo de honor y honestidad, tantas veces manifestado por el pueblo de Sudamérica desde el comienzo de su revolución y que han causado la anarquía y las convulsiones que han desolado al continente en estos últimos treinta años y que desgraciadamente continuarán" (Gómez y Ocaranza, 2011: 686).

Entendemos que en esta última reflexión pesaron los efectos del denominado "temblor grande", evento destructor que se traslapó con una crisis política, económica y social que detonó la dimisión del libertador chileno, constituyendo este fenómeno sísmico parte de un verdadero "concierto catastrófico" mayor, devenido en crisis estructural.

\section{Cuando el terremoto fue noticia}

El destructor evento sísmico del 19 de noviembre de 1822 no solo conmocionó a la incipiente sociedad chilena decimonónica; sino también, y gracias a la "masificación" de los medios de prensa (debido al desarrollo de la imprenta), alcanzó proporciones nunca antes vistas para un asunto que, hasta entonces, solo era tratado de manera local o regional. En este sentido, el contexto sociopolítico en el que se desencadenó, unido a las consecuencias catastróficas que infligió en las ciudades mencionadas y que, por cierto, eran las más densamente pobladas en el Chile de aquel entonces ${ }^{10}$, se conjugaron para constituirla como una noticia de importancia pocas veces vista, alcanzando una difusión notable en diversos medios nacionales y extranjeros.

A este respecto, cabe destacar que la divulgación de este particular terremoto no solo se diferenció de acontecimientos pasados por la profusión en los incipientes medios escritos, también implicó un giro en el tratamiento de este tipo de eventos, por cuanto se comienza a explicar cada vez más como un fenómeno de la naturaleza. Sin embargo, ello no significó la superación de la interpretación mítico-religiosa que había dominado hasta entonces, pero demuestra un tránsito hacia la naturalización del fenómeno, combinando ingredientes científicos y religiosos. En consecuencia, parte de las noticias que aquí transcribimos fueron apareciendo en forma gradual y, en el caso de

10 Por ejemplo, según el censo de 1835, que dio para la República un total de 1.010 .336 habitantes (sin contar a la raza indígena), Santiago, incluido el entonces departamento de Valparaíso, tenía un total de 243.929 habitantes, el equivalente al 24\% de toda la población país (Sotomayor, 1875, 471). 
lugares más alejados, se reiteró la información publicada en medios locales como lo expondremos en esta selección. ${ }^{11}$

\section{La noticia descrita desde el epicentro}

\section{El Cosmopolita, Santiago de Chile, 23 de noviembre de 1822}

"[p. 9] El 19 de noviembre será memorable en los anales de los terribles terremotos terrestres en nuestro hemisferio. A las diez y tres cuartos de la noche después de un calor sofocante se sintió un temblor cual no se había experimentado en este país desde el año de 1730, precedido de un estruendo formidable; y verificándose su explosión por dos violentísimas conmociones que durarían como unos tres minutos, amagando la total ruina de la capital. Su acción terrible ha sido en la opinión de algunos trepidatoria, y en la de otros ondulatoria; y su dirección más verosímil N. O. S. E. Las vibraciones de la tierra continuaron y a eso de las tres de la mañana del 20, hubo un temblor sin ruido sensible y con poca sacudida. Casi media hora después, se vio un meteoro, semejante a una conflagración, que produjo instantáneamente la claridad del crepúsculo de la mañana, y como a las cinco y media de esta, se sintió otro temblor ruidoso, aunque con movimiento lento. El 21 ha habido dos temblores, uno de 4 a 5 de la mañana y otro poco después de las once de esta, ambos sin fuerte conmoción. El 22 dos fuertes temblores, el primero a eso de las 4 de la mañana, y el otro de las 8 a 9 de ella, este último con violento remezón. El 23, otro ligero temblor como a las 8 de la mañana; y hasta las dos de la tarde se han seguido algunas débiles vibraciones. Como a las 4 de la misma, otra bastante sensible, y a las 9 de la noche una de corta entidad. El 24 ha habido algunas vibraciones poco perceptibles.

Estos son los aspectos del terremoto vistos en la capital, donde la consternación ha presentado el cuadro de la calamidad pública más espantosa por los clamores pavorosos y de compunción que resonaban en la población. Los edificios de más elevación han recibido bastante daño, particularmente la catedral, la Merced, san Agustín, el palacio directoral, torres de las cajas y cárcel, casa de moneda, etc....

Según las noticias recibidas hasta ahora, los mayores estragos se han experimentado en Valparaíso, siendo casi total la ruina de las Iglesias y casas, en que se han sepultado una porción de víctimas. La mano protectora de la Providencia salvó la vida del excelentísimo señor director supremo don

11 En la medida de lo posible, se modernizó la grafía y se desplegaron algunas abreviaturas. Del mismo modo, en cada noticia transcrita se hace notar el número de página para facilitar su citación. 
Bernardo O'Higgins, verificándose el desplome de la habitación del fuerte donde se hospedó, instantáneamente después de su evasión de ella. Casa Blanca está por tierra y se cuentan muy pocas personas sacrificadas. Se habla con variedad de la ruina de Melipilla, Aconcagua y otros puntos..."

Gaceta Ministerial de Chile, Santiago de Chile, 27 de noviembre de 1822

"[p. 313] Ayer [19 de noviembre] a las diez y tres cuartos de la noche fue plagado este pueblo con un terremoto tan extraordinario que en obra de dos o tres minutos, que duraría el máximum de su espantosa violencia, se desplomaron o quedaron ruinosos todos sus edificios, sin exceptuarse templo ni casa alguna pública o particular: el mar entretanto se balanceó por la distancia de más de doce pies de elevación: a consecuencia fue declinando el terremoto pero no cesó un solo instante el movimiento de la tierra, bien que remiso, hasta las cuatro y media de la mañana, desde cuya hora se han observado hasta el momento en que escribo, que se repiten los temblores más o menos recios por intervalos de cinco a seis minutos, de modo que puede decirse que se alcanzan sus vibraciones unas a otras; sin embargo, a pesar de la ruina tan terrible, se ha salvado felizmente toda la población sobre los cerros, donde hoy se acampa exceptuando quince o veinte persona entre algunos soldados niños y mujeres, que sabemos haber sepultado las ruinas; bien es que la confusión impide fijar el número de las víctimas que, con grande probabilidad, es mucho mayor; pero puedo asegurar de positivo que ningún empleado público ni hombre visible ha perecido.

Mas, lo que en medio de conflicto tan amargo avivará con mayor fuerza mi corazón, es la idea de los funestos estragos que el terremoto puede haber producido en esa gran capital y demás pueblos del Estado: con ansias y temores profundos he aguardado todo este día noticias acerca de ella, y no habiendo tenido alguna, espero que el gobierno delegado me las participe muy prolijas y circunstanciadas sin pérdida de instantes".

\section{El Mercurio de Chile, Santiago de Chile, 2 de diciembre de 1822}

"[p. 323] A las 10 horas y 54 minutos de la noche se sintió un temblor espantoso que duró dos minutos y medio. En la capital no causó daño alguno digno de consideración, pero fuera de ella los estragos y pérdidas fueron lamentables. Valparaíso, Quillota, la Ligua, Casablanca, fueron enteramente arruinados. Han caído las casas y cercas de un gran número de haciendas y chácaras. Parece que el número de muertos no pasará de doscientos. Aun no sabes a cuantos millones montarán las pérdidas, y no conocemos aún la extensión de los males y ruinas. 
El terremoto no fue precedido de ruido alguno subterráneo: ha sido seguido de emisiones eléctricas del volcán de la cordillera, lo que indica que el peligro ha cesado, y que fue causado por una tempestad subterránea, probablemente originada de la inflamación de [una] inmensa cantidad de hidrógeno. Como el país padece cada siglo de un temblor ruinoso, debemos rendir infinitas acciones de gracias a la Divina Providencia que nos ha librado, y nos inspira dulces esperanzas para todo el siglo 19. Han seguido conmociones ligeras, como sucede en todos los grandes terremotos, y que suelen durar muchos meses. Las habitaciones altas han sido poco conmovidas; y como el terreno de la capital se eleva sobra el nivel del mar como seiscientas varas, conjeturamos que el camino que siguió el gas inflamado, está a inmensa profundidad o distancia de la superficie. Además los temblores no son frecuentes como podían ser por la inmensa abundancia de metales preciosos y de azufre, de sulfates, de [p. 324] hulla, de hierro, etc., que encierra en sus entrañas el opulento Chile. Por ahora desear que se examine mejor el estado de las obras públicas, que se derriben las torres tanto las ruinosas como las no ruinosas pues son siempre amenazantes: que se separen las tejas movidas; que los caminos se reparen, y que los infelices de Valparaíso, etc., sean auxiliados".

\section{La noticia divulgada en el extranjero}

\section{El Argos de Buenos Aires, Buenos Aires, 21 de diciembre de 1822}

"[p. 396] En la noche del 19 del corriente sucedió uno de aquellos fenómenos terribles, con que la naturaleza impone a la imaginación más reposada. En medio de una atmósfera despejada y serena se sintió a las 11 menos 2 minutos un temblor de tierra, que continuó hasta cerca de las 11 y 3 minutos, así su duración ha sido como de 4 minutos. El sacudimiento era tan fuerte, que no nos podíamos sostener de pie: se oyó un susurro, como el que se percibe a la distancia, cuando ruedan algunos coches a la vez, el movimiento fue continuo, y como el que se percibe a la distancia, cuando ruedan algunos coches a la vez, el movimiento fue continuo, y como una especie de ondulación semejante a la de las olas, seguramente a esto debemos la fortuna de no haber experimentado alguna ruina: se ha desplomado uno u otro tapial, y aún se dice de que a distancia de más de una legua al Este, a las 12, y después se han sentido hasta tres veces nuevos remezones, y el día siguiente a la 1 del día.

Todos los signos precursores de estos acontecimientos, ya los habíamos notado: el año lluvioso, celajes muy densos hacia la Cordillera, y algunas nubes de tierra, sobre todo las muchas exhalaciones, o estrella cadentes: observamos uno de estos meteoros como a las 12 de la noche del 4 de este 
mes que iluminó de tal modo la atmósfera, que se oscurecieron del todo las estrellas por cerca de medio minuto, y luego quedó al lado del S.O., o de la cordillera una faja blanquecina, cuya longitud sería como de 12' su disminución fue muy lenta hasta que se formó un círculo semejante a la mayor de las manchas del Sud en aspecto, y magnitud: este meteoro duró como 10 minutos. Por sujetos que acaban de llegar de la República de Chile, sabemos que el director O'Higgins se hallaba en Valparaíso, donde había ido con el objeto de pagar la escuadra, y aun se dice que de licenciarla. El Lord Cochrane vino de Valparaíso a la capital con esta solicitud, y el principal fundamento que alegaba en su apoyo era, que la marinería estaba alzada, y que él no podría contenerla, porque había mucho tiempo que no se les pagaba. El director ha tenido que ceder a este modo de empeñarse. Con motivo de la demora del director en el puerto, el general San Martín había ido a buscarlo desde la capital". ${ }^{12}$

\section{La Abeja Argentina, Buenos Aires, 15 de enero de 1823}

"[p. 35] La naturaleza presenta de tiempo en tiempo los fenómenos más terribles; y en este precioso país se han visto sus [p. 36] sus desgraciados efectos por repetidas ocasiones, pues se cuenta que cada 25 o 30 años se experimenta un terremoto, y el día citado [19 de noviembre] a las 11 menos 5 minutos de la noche siendo claro y sereno el tiempo, el viento en calma, el barómetro en $23^{\circ}$ y el termómetro en $70^{\circ} \mathrm{m}$ y el color de la luna algo pálido pero el de las estrellas lucientes, se oyó de improviso un ruido subterráneo espantoso y al mismo tiempo principió a conmoverse la tierra violenta e impetuosamente de una manera ondular, cuya duración sería de dos minutos y medio, después de este término declinó una vibración suave y continua haciéndose advertir con débiles remezones hasta las 3 y 4 de mañana del 20, que estando el barómetro a los $28^{\circ} 21 / 4$ y con termómetro a los 68 sufrimos un segundo temblor de dos fuertes remezones aunque no comprables con los dichos arriba, cuya duración sería de 6". El cielo despejado y las estrellas más brillantes. En ese momento se vio correr un meteoro de norte a sur, siendo de notar que los movimientos de la tierra venían en dirección N. E. a S. O. y que alumbró tanto como el crepúsculo, tardado su carrera 8". Las oscilaciones parecía que se hubiesen concluido, pero en mi concepto la tierra no estaba quieta; así me lo demostraban el movimiento de un florete que tenía clavado en el suelo. A las 3 y 32 minutos, el barómetro en $28^{\circ} 2$ y el termómetro en $68^{\circ}$ sufrimos otro temblor de un solo remezón menos fuerte que los anteriores de 3 a $4 "$.

12 Esta misma noticia fue reproducida de manera íntegra en El Universal, Madrid, 14-V-1823. 
Efectos causados por el temblor en la capital. Felizmente los primeros movimientos fueron por ondulación y así es que todo el daño principal se ha experimentado en los techos que empezaron a despedir tejas. No hay un edificio en la ciudad que no esté sentido, principalmente los templos. Las dos torres de la iglesia de la Merced se partieron por los cuatro arcos de los campanarios: las perillas colocadas en los ángulos superiores cayeron al medio de la plazuela con todas las tejas de las dos aletas de la puerta principal. El frontis de la iglesia de san Agustín en los dos costados. La torre de san Francisco está ladeada al lado de la iglesia. La capilla de la Estampa se venció por el techo y la fachada sufrió mucho. La catedral ha padecido bastante. Todas las perillas del parapeto del palacio del director cayeron a la plaza. El zaguán de la casa de don Tomás Vicuña se desplomó [p. 39]; también han caído algunos tabiques en varias casas. Son muy notable los daños que hizo en temblor grande en los suburbios siendo de extrañar que algunos edificios nuevos hayan padecido más que los viejos. En la campaña fue generalmente ruinoso en todo el lado norte con particularidad. La hacienda de Polpaico de doña Mercedes Rozas fue destruida en todos sus edificios: la tierra se abrió en muchas partes, y se asegura que de algunas salió agua. Muchas casas de las haciendas del norte se desplomaron y sucedió lo mismo que en Polpaico. Las de Colina se incluyeron en el número. La ciudad de San Felipe en Aconcagua fue casi demolida enteramente, el edifico que ha quedado parado está inhabitable. La villa de los Andes corrió la misma suerte. Putaendo y Quillota por el tenor.

De los lados del sur no se oye decir cosa de consecuencia; parece que allí fue más benigno. En Melipilla sufrieron bastante. La hacienda de Guachur [sic] de doña Mercedes Guzmán quedó sin edificios. En el Mostazal, hacienda de doña Manuelita Santelices, cayó el mirador de las casas, y estas quedaron tan arruinadas que se teme se demuelan por sí de un momento a otro. Las casas de la hacienda de la campaña (antes pertenecientes al conde de la Conquista) se desplomaron. En Rancagua no se han experimentado daños de consideración, pues sólo han padecido los tejados. Del otro lado del Cachapoal no ha sido tan terrible; sin embargo, en algunas partes se abrió la tierra y se dice que también corrió agua por estas grietas como una espuma colorada: este accidente en mi opinión trae su origen de los parajes gredosos o tierras del color indicado, que abundan en aquellos lugares del reino. D. N. Caballero, ingeniero de esta ciudad, fue nombrado por el cabildo para reconocer los edificios públicos; y en consecuencia ha fallado porque todos están malos, particularmente el palacio episcopal y la casa de comedias.

Tengo varias cartas de Valparaíso escritas por personas de juicio y nada asustadas; todas están conformes. La una de ellas y que me merece concepto dice a la letra como sigue: El temblor sería como a las 10 y media de la noche, su movimiento ondulatorio duraría por 4 minutos. Su dirección de Norte a 
Sur, su estrago violento, por que empezar a temblar y los edificios a caer fue uno. En el Almendral no [p. 40] han quedado dos casas servibles, y lo que llaman Puerto se computan 40 existentes, aunque no todas en estado de habitarse. Los movimientos de la tierra continuaron con frecuencia unos más fuertes que otros; no dejaban el intervalo de un cuarto de hora sin dejarse sentir. Esto duraría así hasta las 4 de la mañana del día siguiente, desde cuya hora empezaron a declinar y sucesivamente han ido minorándose siendo el día de hoy en el que menos se han experimentado, pues solo contamos dos, quien sabe si vendrán algunos más. Entre los muertos no hay gente visible: una señora esposa de don N. Berenguel y su sobrina son de las que no se anuncian. En cuanto al número se habla con mucha variedad, pues desde el del 53 hay quien afirma el de 181, y por lo que hace al meteoro que se observó en esa a las cuatro de la mañana creo haya equivocación en la hora porque aquí lo veríamos como a las 3 y cuarto según reloj. Yo me hallaba en uno de los cerros de este puerto y quizás de los menos asustados, bien molesto, sino por los temblores cuanto por los fanatismos de estas gentes. Es muy cierto que la tierra abrió sus grietas en algunos lugares arenosos o flojos, y aunque en algunos cerros se observó lo mismo y los he visto, pero ha sido en la parte inferior del ángulo donde concurre mucha porción de tierra. La mar no ha hecho mayor demostración; se retiró muy poco y volvió a llenar la parte que había vaciado.

Don Onofre Bunster que se hallaba en Valparaíso en la noche del temblor, me ha dicho que habiéndole tomado paseándose en la playa al frente de su casa, quiso subir al cerro inmediato, pero que no pudo conseguirlo por el mucho polvo y piedras que caían, por cuyo motivo se volvió al resguardo a tomar bote, para embarcarse, lo que con trabajo pudo conseguir. Así que se vio a bordo de su buque [y] se propuso observar los movimientos del mar, y al efecto tiró la sondaleza y encontró 13 brazas de agua; al poco rato hizo la misma operación y solo había ocho brazas, en esta alternativa de acceso y retroceso permaneció toda la noche sin causar en tierra el menor daño".

\section{Niles Weekly Register, Baltimore, 8 de marzo de 1823}

"[p. 16] On the nigt of the $18^{\text {th }}$ of Nov. last, all Chili is said to have been shaken to its foundations by an earthquake. Very few houses at Valparaiso were left standing or so littje injured as to be tenantable. The people were living in tents. About three hundred had been taken out of the ruins, and every day other bodies were found! To add to this calamity, wich seems to have been gerenal, a famine was expected -provisions were selling at the most exorbitant prices.

A letter dated Valparaíso, Nov. 25, says -"I was siting with some friends in the my room when the first thing i heard was the falling of the roof - and, on rushing forwards, I found it impossible to stand, the earth was in such 
violent agitation! I fortunetaly got into the street before the house fell- the next moment the earth was rent asunder, leaving a tremendous chasm. The objects on all sides, the screams of the dying and of the fugitive, and the danger wich surrounded me, filled my mind with the most awful emotions. At length I was delivered from danger by the interposition of one of my friends- and I have been living ever since on board ship. Every two or three hours there is a new convulsion of the earth, wich communicates itself to the vessels in the harbour".

\section{El Universal, Madrid, 15 de mayo de 1823}

"[p. 1] El 19 de noviembre quedó Valparaíso destruido por un fuerte temblor de tierra. El número de los que perecieron fue comparativamente corto, pues según pudo averiguarse al día siguiente, para que el supremo director lo pusiese en noticia de las autoridades de Chile, hubo de 15 a 20 muertos. En Santiago se resintieron algunos edificios".

\section{Gaceta de Madrid, Madrid, 15 de mayo de 1823}

"[p. 31] Santiago de Chile 9 de diciembre. El 19 del pasado a las 9 de la noche un terrible terremoto sobrevino de repente sin ruido, pero con un movimiento de tierra espantoso, causando muchos estragos, particularmente en Valparaíso, donde además de haber echado por tierra casi todos sus edificios, han quedado sepultadas entre sus ruinas más de 300 personas, corriendo la misma suerte Casablanca, Quillota, Melipilla y otros. Esta capital ha sufrido menos, y sin embargo han caído algunas iglesias, muchas trizadas; y los edificios de casa de moneda, aduana, consulados, cajas, cárcel y de comedia no se pueden habitar sin grandes reparos, para lo cual se ha nombrado un ingeniero, cuya comisión se extiende también a las casas particulares, porque son infinitas las necesitadas de estos reparos. Yo hace cuatro días que dejé mi alojamiento campestre, cuyo techo ha sido una higuera, y casi toda la ciudad ha abandonado sus antiguos hogares por temor de que la repetición de más de 150 temblores que se han seguido al primero, de menos duración y estrépito, causasen los mismos estragos que nos anuncian de otras partes. En fin la Providencia divina nos ha mirado con ojos de piedad, y vamos poco a poco recuperando la tranquilidad perdida. La duración del gran temblor pasó de 3 minutos".

\section{Niles Weekly Register, Baltimore, 17 de mayo de 1823}

"[p. 171] Earthquakes.at Valparaiso. Extract of a letter from a Bostonian, residing at Valparaíso, to a gentleman in Boston, dated November 26 ${ }^{\text {th }}, 1823$. The present is principally to acquaint you of the effects of a most tremendous and terrible earthquake, wich was experienced here on the evening of the $18^{\text {th }}$. It took place between 10 and 11 o'clock, and the first shock, 
which was propably of two or three minutes duration, laid the greater part of Valparaiso in ruins, and spread consternation and terror every where about. Several other heavy shocks followed: but not to be compared with the first, or to do so much injury. They continued very frequent through the night, and every one fled to the hills and shipping for safety. Many lives were lost by the fall of buildings, but the number has not been ascertained; and nearly 200 are known to have perished and others are missing; many too were wounded severely, among whom was the supreme director, who was down at that time from the city, and who very narrowly escaped while the governor's palace was trembling over his head, This building is so entirely in ruins that it will be required to rebuild it from the foundation. The churches are, some of them, levelled to the ground, and others so rent and shattered as to ruin them. The custom house buildings are injured very much, and, in short, there is scarce a building here wich has not received more or less damage. Indeed, there is not a dozen houses in the place at this time, that would be considered habitable with safety and comfort. Several light shocks have been left every day and night since the first, and fears are entertained that something more terrible will follow.

Earthquakes are frequent in this country, and there is never a year without them; but they are not often very heavy, and it is nearly a century since they have experienced one so dreadful as at this time. Some of the neighboring towns and villages are entirely ruined, and there was the most painful apprehensions respecting the fate of the capita, (Santiago), but fortunately that city has escaped with comparative trifling injury. If it had been felt as severe there as in this place, it must have put a stop to business for a considerable time to come. As it is, there has been a total suspension for the week past, and it had been resumed only a day or two before, after waiting two months for the new commercial reglamento. On the $18^{\text {th }}$ we had been removing to another building, and the goods, furniture, \&c. were piled up loose and promiscuously about the room where we slept, not having time to stow them away; we were in bed before the shock came on, and the lights were all extinguished; here we were on a second floor, high from the street, and unacquainted with the stairs and passage out. I will not attempt to describe the horror of the moment of the earthquake; the noise was like a long peal of thunder; the floor of bricks under us, rattled; the tenders over our heads cracked; the lime, and dirt from the mud walls almost suffocated us, while the house rolled and trembled like a ship in a heavy short sea. We escaped in safety to the streets and there the tiles, \&c. were falling from the roofs, and though the night was beautifully clear and calm before, such clouds of dust arose from the trembling hills and falling walls, that it was difficult to see or breathe. The screams and cries of women and children were heard in every direction, and people of all classes were running almost naked through the streets, calling on saints for mercy and protection. 
Nearly the whole population are now scattered about the hills around the port, in tents; and it is said, that most of the inhabitants of Santiago have also left their houses and gone into the fields. The English families, as also our consuls, have all embarked on board the shipping, not more for safety than because their dwellings are rendered unfit to inhabit.

The loss or damage of goods, furniture, \&c. I believe has been much less than was anticipated. Indeed, I hear of nothing considerable; but these are greatly exposed to robberies, and, though guards are kept in the streets, it is necessary for every one to go armed and look out for his own property. I am happy to say that none of our countrymen have lost their lives by this disaster; nor have I heard of any other foreigner who is much known. It is a remarkable fact, which must be interesting to the inquiries of the curious, that on the day preceding this tremendous shock, there were myriads of fished seen dead and dying on the water along shore. Capt. Scott, of the Ida, who has since arrived here, was that evening on shore at St. Antonia [sic], a little port about 30 miles to the $\mathrm{S}$. where the shock was most terrible; and next morning, going off to his ship, rowed through the floating fishes, with which he says he could have filled his boat in a few minutes. I have read, or heard before, of similar instances, and this corroboration leaves no doubt that the destruction was occasioned [p. 172] by the earthquake; but it would be difficult to determine in what manner it produces such an effect."

\section{Referencias bibliográficas}

\section{a) Publicaciones oficiales}

Constitución Política del Estado de Chile (1822). Santiago de Chile: Imprenta del Estado.

Proyecto de Constitución Provisoria para el Estado de Chile (1818). Santiago de Chile: Imprenta del Gobierno.

\section{b) Periódicos y revistas}

El Argos de Buenos Aires, Buenos Aires, 21-XII-1822

El Cosmopolita, Santiago de Chile, 23-XI-1822

El Mercurio de Chile, Santiago de Chile, 2-XII-1822

El Universal, Madrid, 14, 15-V-1823

Gaceta de Madrid, Madrid, 14 y 15-V-1823

Gaceta Ministerial de Chile, Santiago de Chile, 27-XI-1822 
La Abeja Argentina, Buenos Aires, 15-I-1823

La Aurora de Chile, Santiago de Chile, 28-I-1813

Niles Weekly Register, Baltimore, 8-III-1823 y 3-V-1823

\section{c) Artículos de revistas y capítulos de libros}

Alberola, A. (2005). "El terremoto de Lisboa en el context del catastrofismo natural en la primera mitad del siglo XVIII", en Cuadernos Dieciochistas. № 6 , pp. 19-42.

Alberola A. (2009). "De la percepción popular a la reflexión erudita. La transmisión de la cultura de la catástrofe en la España del siglo XVIII", en La réception de la cultura de masses et des cultures populaires en Espagne (XVIIIe-XXe siècles). Paris: Université de la Sorbonne Nouvelle de Paris, pp. 39-67.

Barrientos, S. (2007). "Earthquakes in Chile", en T. Moreno y W. Gibbons, The Geology of Chile. Londres: The Geological Society of London, pp. 263-287.

Cid, G. (2014). "¿Castigo divino o fenómeno natural? Mentalidad religiosa y mentalidad científica en Chile en torno al terremoto de 1822", en Revista de Historia y Geografía, № 30. Santiago: Universidad Católica Silva Henríquez, pp. 85-109.

Goldman, N. (2003). "Casos de continuidad y ruptura: Virreinato del Río de La Plata y Capitanía General de Chile, 1810-1830", en G. Carrera, Historia general de América Latina, vol. V, pp. 185-206.

Gorigoitía, N. (2016). "El proceso de independencia de Chile y la coyuntura del terremoto de 1822: Un concierto catastrófico", en M. Chust, El Sur en Revolución. La insurgencia en el Río de la Plata, Chile y el Alto Perú. Castellón de la Plana: Ediciones de la Universidad Jaume I, pp. 187-210.

Lomnitz, C. (2004). "Major earthquakes of Chile: a historical survey, 1535-1960", en Seismological Research Letters, № 75, pp. 368-378.

Miquel, J. (1859). "Apuntes sobre el terremoto de 1822", en Anales de la Universidad de Chile, tomo XVI, pp. 233-282.

Palacios, A. (2010). "Cotidianeidad y religiosidad frente a la catástrofe: el terremoto de 1822", en M. Sánchez, Historia de la Iglesia en Chile: la iglesia en los tiempos de la Independencia. Santiago de Chile: Editorial Universitaria, pp. 371-399.

Thurn, C. (1919). "Descripción de los estragos que causó en Valparaíso el terremoto del 19 de noviembre de 1822", en Revista Chilena de Historia y Geografía, tomo XXI, pp. 189-193. 


\section{d) Libros}

Amunátegui, M. (1855). La dictadura de O'Higgins. Santiago de Chile: Imprenta de Julio Belin.

Barros Arana, D. (1857). Historia general de la Independencia de Chile. Santiago de Chile: Imprenta del Ferrocarril, tomo III.

Barros Arana, D. (1984). Historia general de Chile. Santiago de Chile: Rafael Jover editor, tomo XIII.

Cartes, A. (2014). Un gobierno de los pueblos: relaciones provinciales en la independencia de Chile. Valparaíso: Ediciones Universitarias de Valparaíso.

Cochrane, T. (1863). Memorias de Lord Cochrane, conde de Dundonald. París: Imprenta de Eduardo Blot.

Eyzaguirre, J. (1950). O'Higgins. Santiago de Chile: Editorial Zig-Zag.

Famín, C. (1839). Historia de Chile. Barcelona: Imprenta de Guardia Nacional.

Gendrin, V. (1856). Récit historique, exact et sincere, par mer et par terre, de quatre voyages faits au Brésil, au Chili, dans les cordilleres des Andes, a Mendoza, dans le desert, et a Buenos Aires. Versalles: Imprenta Klefer.

Gómez, A. y F. Ocaranza (2011). Epistolario de don Bernardo O'Higgins Riquelme. Santiago de Chile: Universidad Bernardo O'Higgins.

Graham, M. (1953). Diario de mi residencia en Chile. Santiago de Chile: Editorial del Pacífico (1ºdición, 1824 en inglés).

Longeville, G. (1923). Memorias de un oficial de marina inglés al servicio de Chile durante los años de 1821-1829. Santiago de Chile: Imprenta Universitaria (1 edición, 1831 en inglés).

Matamoros, O. (1994). Análisis de la amenaza de licuefacción, lavas, lahares y caída de cenizas volcánicas. Costa Rica; sin editorial.

Montessus de Ballore, F. (1912). Historia sísmica de los Andes Meridionales. Santiago de Chile: Imprenta Cervantes, tomo IV.

O'Phelan, S. (2010). Bernardo O'Higgins y sus estancias en el Perú. Lima: Fondo Editorial del Congreso del Perú.

Palacios, A. (2015). Entre ruinas y escombros: los terremotos en Chile durante los siglos XVI al XIX. Valparaíso: Ediciones Universitarias de Valparaíso.

Palacios, A. (2016). Historia ilustrada de los megaterremotos ocurridos en Chile entre 1647 y 1906. Valparaíso: Ediciones Universitarias de Valparaíso. 
Proctor, R. (1920). Narraciones del viaje por la cordillera de los Andes y residencia en Lima y otras partes del Perú en los años 1823 y 1824. Buenos Aires: Vaccarro, (1edición, 1825 en inglés).

Riquelme, D. (1899). Compendio de historia de Chile. Valparaíso: Litografía e Imprenta Sud-Americana de Barra.

Schmidtmeyer, P. (2014). Viaje a Chile a través de Los Andes. Buenos Aires: Editorial Claridad (1edición, 1824 en inglés).

Sepúlveda, A. (2007). Bernardo: una biografía de Bernardo O'Higgins. Santiago de Chile: Ediciones B.

Sotomayor, R. (1875). Historia de Chile durante los cuarenta años trascurridos desde 1831 hasta 1871. Santiago de Chile: Imprenta de la Estrella de Chile.

Vicuña Mackenna, B. (1860). El ostracismo del general O'Higgins. Valparaíso: Imprenta y Librería del Mercurio de Santos Tornero.

V. AA. (2016). El "efecto 1812" en la prensa y la ciencia del siglo XIX. Caracas: Academia Nacional de la Historia. 